\title{
Sarcoma fibroblástico mixoinflamatorio acral: caso clínico y revisión bibliográfica
}

\author{
A. García Jiménez, I. Proubasta, N. Arriaga, I. Gracia Alegría \\ Servicio de Cirugía Ortopédica y Traumatología. Hospital de la Santa Creu i Sant Pau. \\ Universitat Autònoma de Barcelona. Barcelona. España.
}

Resumen: El sarcoma fibroblástico mixoinflamatorio es un tumor maligno de bajo grado de partes blandas, generalmente localizado en extremidades y que afecta a sujetos de mediana edad. Clínicamente, la lesión es infiltrativa, indolora y de curso evolutivo lento. Se presenta un caso que afecta la mano, haciendo una extensa revisión de la bibliografía, con especial atención a su diagnóstico y tratamiento.

Palabras claves: Sarcoma, fibroblástico, mixoinflamatorio, mixoide, inflamatorio, acral.

\section{Acral Myxoinflammatory Fibroblastic Sarcoma: Case Report and Review of the Literature}

\begin{abstract}
Acral myxoinflammatory fibroblastic sarcoma is a rare low-grade malignant soft tissue tumor, usually observed in the extremities of middle-aged patients. Clinically, the lesion is infiltrating, pain-free and a slow progression. A case involving the hand is presented, doing an extensive review of the literatura, with particular attention to diagnosis and treatment.
\end{abstract}

Key words: Sarcoma, fibroblastic, mysoinflammatory, myxoid, inflammatory, acral.

El sarcoma fibroblástico mixoinflamatorio (SFMI) es un sarcoma de partes blandas muy poco común, de tipo multinodular y que se localiza con preferencia distalmente, motivo por el cual se añade el apelativo "acral" cuando asienta en manos y/o pies'. El tratamiento de elección es el quirúrgico, mediante la exéresis de la tumoración. Aunque dicho tumor suele ser de bajo grado, en ocasiones puede producir metástasis. Sin embargo, su principal problema es su alta incidencia de recidiva local ${ }^{2}$.

El motivo del presente trabajo es describir un nuevo caso de SFMI, localizado en mano, y cuyo diagnóstico solo pudo determinarse después de su estudio anatomopatológico tras la resección quirúrgica. A partir de este caso, se realiza una extensa revisión sobre esta particular patología, con especial atención a su diagnóstico y tratamiento.

\section{CASO CLÍNICO}

Se trata de un varón de 31 años de edad, que acudió derivado de otro centro para estudio y tratamiento de una tumoración en la mano derecha de dos años de evolución. Según refiere, notó la aparición de una pequeña masa en la cara palmar de la mano, entre los dedos anular y meñique, de localización digitopalmar, indolora, y que fue aumentando de tamaño progresivamente en los últimos seis meses. Niega antecedente traumático.

El examen físico reveló la presencia de una gran tumoración en la palma de la mano derecha, de consistencia blanda y que se extendía hacia la cara dorsal entre los espacios intermetacarpianos tercero y cuarto (-) Figura I. Esta tumoración no era dolorosa a la palpación y no presentaba signos inflamatorios. Sin embargo, existía un déficit de flexión activa de los dedos meñique y anular, al existir un conflicto de espacio por el volumen de la misma.

El estudio radiográfico simple fue normal y en la Resonancia Magnética (RM) se objetivó un proceso expansivo de partes blandes, situado en la profundidad de la aponeurosis palmar, que infiltraba la musculatura hipotenar y los músculos lumbricales de la mano, extendiéndose dorsalmente entre el $4^{\circ}$ y $5^{\circ}$
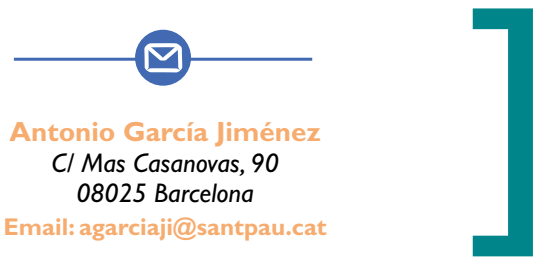


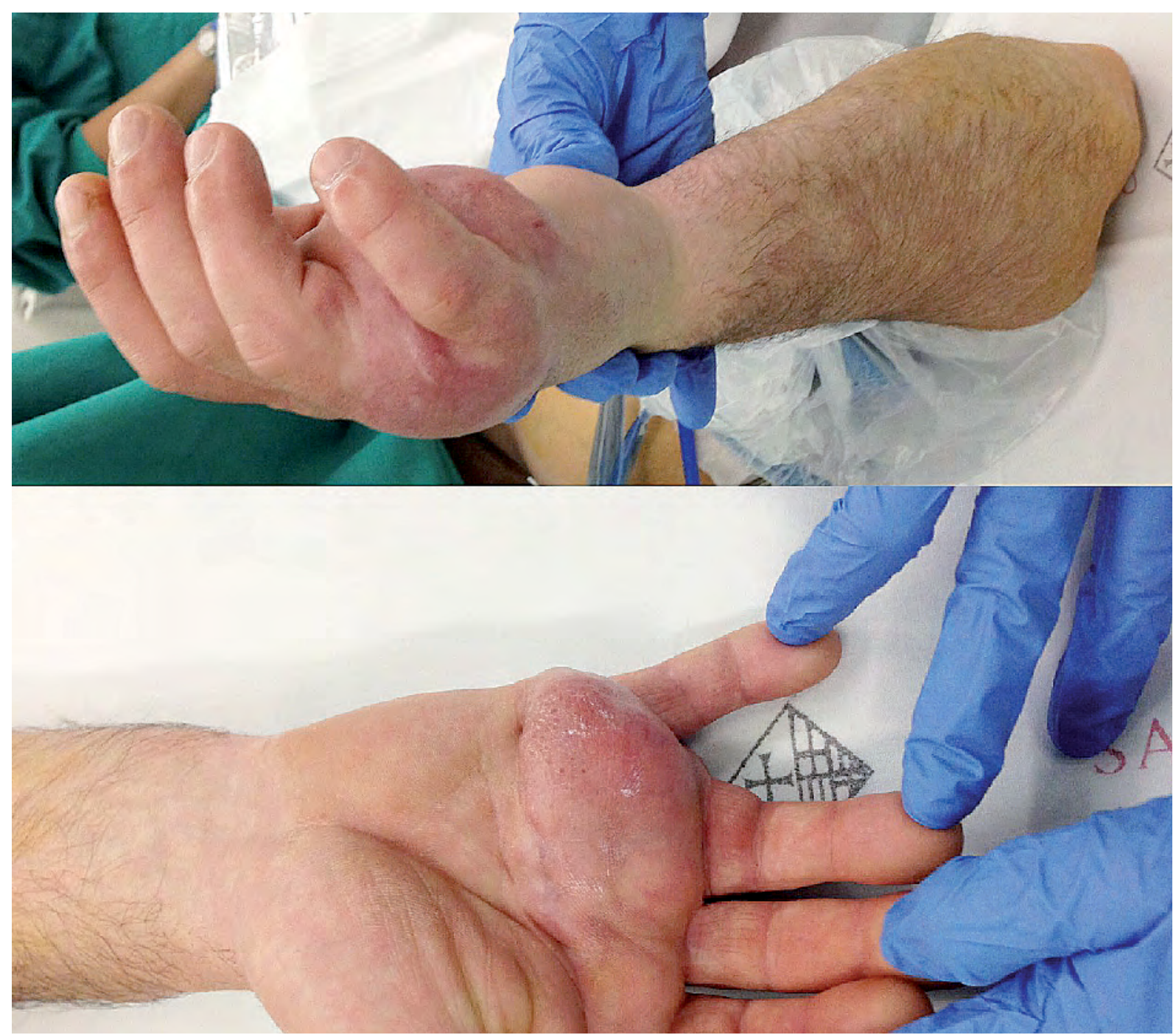

Figura ॥. Imagen clínica prequirúrgica de la mano del paciente.

radios (- Figura 2. Sin un diagnóstico claro, se procedió a la biopsia (por punción-aspiración) con tru-cut de la lesión.

El estudio anatomopatológico mostró la presencia de abundantes vasos de calibre pequeño-mediano y algunas células con vacuolización citoplasmática ("pseudolipoblastos"). El estudio inmunohistoquímico reveló la positividad para CD34 y negatividad para la proteína S-100. Los hallazgos morfológicos, principalmente el estroma mixoide prominente, plantearon el diagnóstico diferencial entre lesiones benignas (mixoma, ganglión o angiomixoma superficial) y, debido al tamaño, con lesiones malignas (sarcoma fibromixoide de bajo grado o liposarcoma mixoide). La negatividad para la proteína S-100 prácticamente descartó un tumor de estirpe neural. Asimismo, el estudio FISH para detección de reordenamiento del gen DDIT3 resultó negativo, lo que no apoyó el diagnóstico diferencial de liposarcoma mixoide. Así pues, sin un diagnóstico histopatológico cierto, se decidió por el tratamiento quirúrgico debido a la rápida progresión y expansión de la lesión.

Bajo anestesia axilar, sin vaciamiento venoso ni uso de isquemia, se realizó un abordaje doble de la tumoración. Así, mediante incisión media longitudinal dorsal de la mano, se continuó por el espacio interdigital entre anular-meñique hasta el pliegue palmar distal. A dicho nivel, se practicó una incisión transversal siguiendo dicho pliegue $y$, a partir de la misma, se extendió la incisión en sentido proximal, en zig-zag, hasta el límite inferior del retináculo flexor. Una vez levantados los colgajos cutáneos correspondientes, se visualizó una gran tumoración palmar sin clara delimi- 

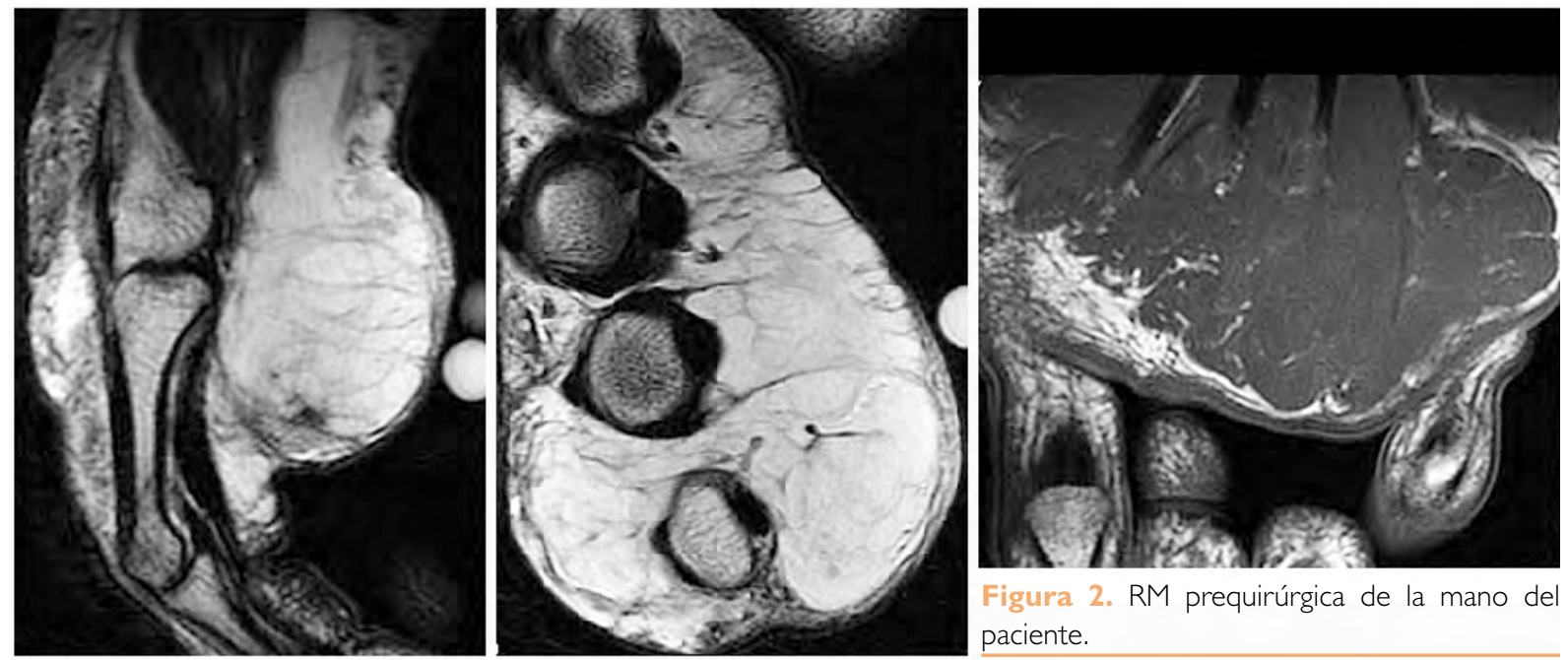

Figura 2. RM prequirúrgica de la mano del paciente.

tación, de aspecto notoriamente mixoide, de tonalidad amarillo-parduzca y distinguiéndose una zona de transición en la región interdigital 4-5, por la cual se continuaba hacia la cara dorsal, por debajo de ligamento intermetacarpiano (a) Figura 3. La tumoración, se encontraba adherida a las vainas tendinosas de los tendones flexores, englobando en nervio digital del $4^{\circ}$ espacio intermetacarpiano. Después de resecar la misma, incluyendo el nervio digital citado, se procedió al cierre de la piel con puntos simples de monofilamento 5/0, y se colocó un yeso antebraquiocarpiano, incluyendo el pulgar. El estudio microscópico de la pieza reveló la presencia de abundantes nidos de células epitelioides y fusiformes atípicas en un estroma mixoide con densos infiltrados inflamatorios, compatibles con SFMI.

Durante los controles posteriores, el paciente mostró una evolución tórpida de los bordes de la herida qui-

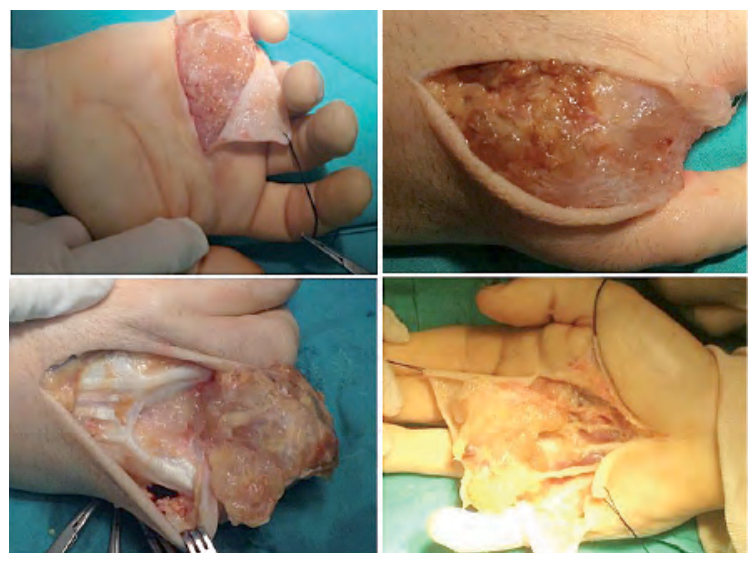

Figura 3. Imagen intraoperatoria de la tumoración. rúrgica, requiriendo la resección de los bordes necróticos en consultas externas de forma ambulatoria. El control por RM a los 3 meses de la cirugía, evidenció pequeños restos tumorales, destacando uno de $7 \mathrm{~mm}$, entre las cabezas del $4^{\circ}$ y $5^{\circ}$ metacarpiano y, otro de $13 \mathrm{~mm}$, en la eminencia hipotenar (2) Figura 4. Se inició tratamiento radioterápico, con un total de 5 sesiones, completando una dosis de 50Gy. Tras las mismas, el paciente desarrolló una radiodermitis del dorso de la mano. A los 4 meses, tras la finalización de la radioterapia, y 8 meses tras la cirugía, se realizó una nueva RM, en la que se observó la desaparición completa de todos los restos tumorales previos, pero con la aparición de una nueva lesión nodular de morfología irregular, localizada subcutáneamente a la altura de la cabeza del segundo metacarpiano, con señal aumentada en T2 y captación del contraste, indicando recidiva tumoral (4) Figura 5, motivo por el cual se realizó su exéresis. Un año después de esta última
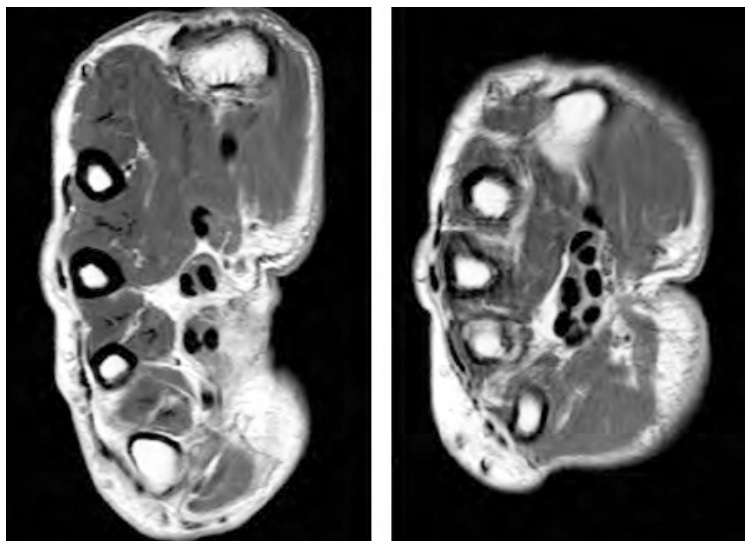

Figura 4. RM tres meses tras la cirugía. 


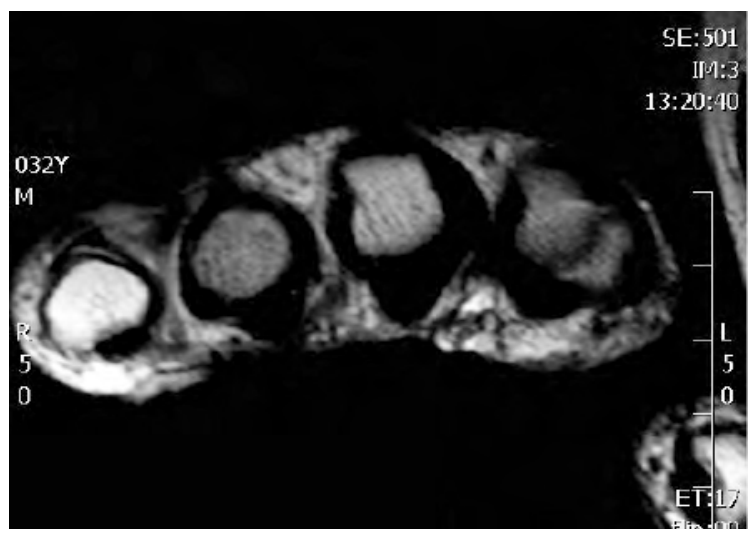

Figura 5. RM cinco meses tras la radioterapia, y 8 meses tras la cirugía.

cirugía, el paciente no ha presentado ninguna recidiva más, ni clínica ni por RM.

\section{DISCUSIÓN}

El SFMI fue descrito simultáneamente por primera vez en 1998 por tres grupos de patólogos: Kindblom², Montgomery ${ }^{3}$ y Michal $^{4}$. Dicho tumor suele desarrollarse en pacientes de todas las edades, aunque especialmente entre la $4^{\mathrm{a}}$ y $5^{\mathrm{a}}$ décadas de la vida. Ambos géneros se encuentran afectados por igual ${ }^{5}$. Su forma de presentación es la de una masa en las extremidades, especialmente dedos, y habitualmente está incluida en el tejido celular subcutáneo, con un crecimiento lento y generalmente indolora ${ }^{6-8}$.

El caso que presentamos se ajusta bastante bien a las características descritas, pues es un paciente en la cuarta década de la vida, tiene una tumoración de dos años de evolución, indolora y en la región distal (acral) de la extremidad superior derecha. Si bien las extremidades son la localización de preferencia, han sido descritos en otros sitios, motivo por el cual la OMS retiró el termino "acral", aunque se les continúa añadiendo este apelativo cuando afecta a manos y/o pies.

Desde el punto de vista clínico, el diagnóstico diferencial debe plantearse con procesos inflamatorios, infecciosos crónicos, y con tumores benignos.

En cuanto a los estudios por la imagen, la RMN no permite un diagnóstico claro, pues las imágenes obtenidas pueden superponerse a otras entidades, como son el tumor de células gigantes de vainas tendinosas, fascitis proliferativa, fibromixoma acral, liposarcoma mixoide y fibrosarcoma mixoide. Sin embargo, es común observar un realce heterogéneo con contraste en áreas donde existe abundante estroma mixoide ${ }^{9,10}$.

Macroscópicamente, el tumor presenta un tamaño variable, entre 1,5 a $18 \mathrm{~cm}$, polilobulado y de color amarillento. En ocasiones, pueden observarse áreas de necrosis y/o hemorragia. A nivel microscópico, presenta tres características: I) arquitectura multinodular, alternando áreas densamente celulares y áreas mixoides hipocelulares; 2) infiltrado mixoide inflamatorio y 3) células gigantes a cuerpo extraño y lipoblásticas ${ }^{|-3,| 1}$. No existe un fenotipo inmunohistoquímico característico, aunque en todos los casos las células tumorales son vimentina positivas. En cuanto a otros marcadores inmunohistoquímicos, destacan la positividad a CD68 en el $66 \%$ de los casos, a CD34 en el 28\%, a actina de músculo liso en el $6 \%$ y a $\mathrm{KL}$ I en el I I\% de los casos ${ }^{\prime \prime}$.

La fisiopatología del SFMI es desconocida. No se han identificado agentes infecciosos que puedan estar implicados y solo en un estudio citogenético de un caso, se encontró un cariotipo complejo con translocación recíproca $\mathrm{t}(\mathrm{l}: 10)(\mathrm{p} 22, \mathrm{q} 24)$ y pérdida de los cromosomas 3 y $13^{12}$.

El tratamiento consiste en la resección tumoral amplia, que puede ser coadyuvada con radioterapia incluso en aquellos pacientes con márgenes positivos ${ }^{\prime 3}$. En este sentido, si bien la radioterapia no es bien recibida en la mano, nuestra experiencia y la de otros autores ${ }^{13}$, es que puede conseguirse una alta tasa de curaciones. De hecho, incluso se recomienda su uso en el preoperatorio para otros muchos sarcomas de partes blandas $^{14}$. Sin embargo, la recidiva local es alta, entre el $22 \%$ y el $67 \%$ de los casos, y se produce de media a los 4- 10 meses, tal como ocurrió en nuestro caso'3,15,16. En algunas ocasiones, se ha tenido que proceder a la amputación por recidivas múltiples locales ${ }^{13,16}$. Sólo se han descrito tres casos de metástasis en la literatura y ninguno de ellos con resultado de muerte asociada al SFMI ${ }^{17}$, aunque es probable que ello pueda suceder al no existir estudios a largo plazo".

\section{CONFLICTOS DE INTERESES}

Los autores declaran no tener conflictos de intereses.

\section{BIBLIOGRAFÍA}

I. Irisarri C, Capomassi M. Tumores malignos de partes blandes. En: Irisarri C, Capomassi M (Eds.). 
Tumores de la Mano. Madrid: Panamericana; 2009, p. 34-5.

2. Meis-Kindblom JM, Kindblom LG. Acral myxoinflammatory fibroblastic sarcoma: a low-grade tumor of the hands and feet. Am J Surg Pathol. 1998;22:9| |-24.

3. Montgomery EA, Devaney KO, Giordano TJ, Weiss SW. Inflammatory myxohyaline tumor of distal extremities with virocyte or Reed-Sternberg-like cell: a distinctive lesion with features simulating inflammatory conditions, Hodgkin's disease, and various sarcomes. Mod Pathol. 1998; 1 1:384-91.

4. Michal M. Inflammatory myxoid tumor of the soft parts with bizarre giant cells. Pathol Res Pract. 1998; 194:529-33.

5. Chahdi H, Damiri A, Oukabli M, Albouzidi A, Boubaid S, Lazrek K. Acral myxoinflammatory fibroblastic sarcoma. Orthop Traumatol Surg Res. 20 10;96: 597-9.

6. Hohendorff B, Kurzen P, Giger A, Stauffer E. Myxoinflammatoric fibroblastic sarcoma in the hand. J Hand Surg EurVol. 2007;32E:359-60.

7. Lang J, Dodd L, Martinez S, Brigman B. Case reports: acral myxoinflammatory fibroblastic sarcoma: a report of five cases and literature review. Clin Orthop. 2006;445:254-60.

8. Leti AA, Pancladi G, Limontini S, Righ RE, Landi A. Su di un raro caso di sarcoma fibroblastico mixoinflammatorio acrale allà mano. Riv Chir Mano. 2002;39:1 85-9.

9. Narvaez JA, Martinez S, Dodd LG, Brigman BE. Acral myxoinflammatory fibroblastic sarcomes: MRI findings in four cases. AJR. 2007; I 88: I 302-5.
10. Tateishi U, Hasegawa T, Onaya H, Satake M, Arai Y, Moriyama N. Myxoinflammatory fibroblastic sarcoma: MR appearence and pathologic correlation. AJR. 2005; 1 84: 1749-53.

I I. Vaquero M. Sarcoma fibroblástico mixoinflamatorio: presentación de un caso y revisión de la literatura. Rev Esp Patol. 2008;41:223-6.

12. Lambert I, Debiec-Rychter M, Guenlinckx P, Hagemeijer A, Sciot R. Acral myxoinflammatory fibroblastic sarcoma with unique clonal chromosomal changes. Virchows Arch. 200 I;438:509- 12.

13. Tejwani A, Kobayashi W, Chen Y-LE, Rosenberg A, Yoon S, Raskin KA,Rosenthal DI, Nielsen GP, Hornicek FJ, DeLaney TF. Management of acral mysoninflammatory fibroblastic sarcoma. Cancer. 2010;1 16:5733-9.

14. Talbert ML, Zagars GK, Sherman NE, Romsdahl $\mathrm{MM}$. Conservative surgery and radiation therapy for soft tissue sarcoma of the wrist, hand ankle, and foot. Cancer 1990;66:2482-91.

15. Silver AG, Baynosa RC, Mahabir RC, Wang WZ, Zamboni WA, Khiabani KT. Acral mysoinflammatory fibroblastic sarcoma: a case report and literatura review. Can J Plast Surg. 2013;21:92-4.

16. Sakaki M, Hirokawa M, Wakatsuki S, Sano T, Endo K, Fujii Y, Ikeda T, Kawaguchi S, Hirose T, Hasegawa T. Acral mysoinflammatory fibroblastic sarcoma: a report of five cases and review of the literatura. Virchows Arch. 2003;442:25-30.

17. Lombardi R, Jovine E, Zanini N, Salone MC, Gambarotti M, Righi A, Balladelli A, Colangeli M, Rocca M. A case of lung metastasis in myxoinflammatory fibroblastic sarcoma: analytical review of one hundred and thirty eight cases. Int Ortop. 20।3;37:2429-36. 